\title{
Os restaurantes por peso no contexto de alimentação saudável fora de casa ${ }^{1}$
}

\author{
Pay-per-kilo restaurants in the context of \\ healthy eating away from home
}

Melina Valério dos SANTOS²

Rossana Pacheco da Costa PROENÇA²

Giovanna Medeiros Rataichesck FIATES ${ }^{2}$

Maria Cristina Marino CALVO $^{3}$

RE S U M O

O consumo de refeições fora de casa é uma tendência em diferentes regiões do mundo, mas há indícios de que essa prática possa apresentar riscos à saúde. É reconhecido que os comportamentos e as escolhas alimentares dos indivíduos são complexos e estão condicionados a diversos fatores. O fator ambiental vem recebendo crescente atenção dentre as variáveis que envolvem as escolhas alimentares. Nesse contexto, este artigo discute a possibilidade de os restaurantes por peso, modalidade de serviço muito utilizada na realidade brasileira, representarem uma alternativa saudável de alimentação fora de casa. Os dados científicos disponíveis relativos aos restaurantes por peso ainda são escassos, não permitindo uma avaliação sistematizada sobre o tema. Assim, com base em pesquisa bibliográfica, discutem-se os modelos praticados e alguns estudos que comparam a possibilidade de escolhas saudáveis em restaurantes com diferentes modalidades de distribuição. Sugere-se que, devido às suas características - que incluem a diversidade, a possibilidade da prática de preços acessíveis, a rapidez e a conveniência -, os restaurantes por peso possam estimular a escolha de alimentos saudáveis nas refeições realizadas fora de casa. Ressalta-se, também, a importância de intervenções nutricionais tanto na disponibilização dos alimentos quanto na orientação à população para escolhas saudáveis nesse contexto.

Termos de indexação: Alimentação coletiva. Comportamento alimentar. Restaurante.

A B S T R A C T

Consumption of food away from home is a trend in different regions of the world, with indications that this practice may pose a health risk. It is recognized that eating behaviors and food choices are complex and conditioned to several factors. The environmental factor has been receiving increasing attention among the

\footnotetext{
1 Artigo elaborado a partir da dissertação de M.V. SANTOS, intitulada "Características sociodemográficas e componentes alimentares dos pratos de comensais em restaurantes por peso". Universidade Federal de Santa Catarina; 2009. Apoio: Coordenação de Aperfeiçoamento de Pessoal de Nível Superior.

2 Universidade Federal de Santa Catarina, Programa de Pós-Graduação em Nutrição, Núcleo de Pesquisa de Nutrição em Produção de Refeições. Campus Universitário, Trindade, 88040-900, Florianópolis, SC, Brasil. Correspondência para/Correspondence to: R.P.C. PROENÇA. E-mail: <rossana@mboxl.ufsc.br>.

3 Universidade Federal de Santa Catarina, Programa de Pós-Graduação em Saúde Pública. Florianópolis, SC, Brasil.
} 
642 M.V. SANTOS et al.

variables that involve food choices. In this context, this article discusses the possibility of pay-per-kilo restaurants, a typical Brazilian service, being a healthy alternative when eating out. Scientific facts related to pay-per-kilo restaurants are scarce, making it unfeasible to perform a systematic assessment of the subject. Hence, this review discusses restaurant modalities and some studies that compare the possibility of healthy choices in restaurants with different distribution modalities. Given its characteristics, which include diversity, reasonable prices, speed and convenience, pay-per-weight restaurants can encourage healthy food choices away from home. Also, there is emphasis on the importance of nutrition interventions that make healthy foods available and educate the population to make healthier food choices.

Indexing terms: Collective feeding. Feeding behavior. Restaurants.

\section{N T R O D U ÇÃ O}

A maior concentração de pessoas nos grandes centros urbanos, associada ao aumento da participação das mulheres no mercado de trabalho e às alterações no estilo de vida da população, vem induzindo mudanças nos padrões de vida e comportamentos alimentares. Algumas dessas alterações são caracterizadas pela falta de tempo para o preparo e consumo dos alimentos, o que resulta em busca por conveniência e em deslocamento das refeições de casa para estabelecimentos comerciais ${ }^{1}$.

Estudos realizados em países desenvolvidos têm mostrado uma associação positiva entre a frequência de consumo de refeições fora de casa, especialmente em restaurantes fast-food, e o aumento de Índice de Massa Corporal (IMC) e ganho de peso ${ }^{2,3}$. Uma possível explicação para tal relação são os elevados teores calóricos, de gorduras totais, gordura saturada e açúcar presentes nos alimentos preparados fora de casa ${ }^{4,5}$.

Em países em desenvolvimento, são poucos os estudos que investigam a associação entre alimentação fora de casa e peso corporal. No Brasil, uma pesquisa de base populacional, de Bezerra \& Sichieri ${ }^{6}$, utilizando dados da Pesquisa de Orçamentos Familiares 2002/037 , observou que a alimentação fora de casa esteve positivamente associada ao sobrepeso e à obesidade entre os homens de áreas urbanas do país, mas não entre as mulheres. Além da escassez de trabalhos sobre o tema, outra limitação comum aos estudos a respeito de alimentação fora de casa é a não distinção das diferentes modalidades de restaurantes $^{8,9}$ ou a análise somente de fast-foods ${ }^{2-4}$.
Considerando que a obesidade é um problema de saúde pública global e diante da possibilidade de a alimentação fora de casa representar um risco para a saúde, este artigo apresenta uma discussão sobre possibilidade de escolhas saudáveis nesse contexto, considerando a alternativa dos restaurantes self-service por peso, modalidade de serviço tipicamente brasileira. Destaca-se que os dados disponíveis relativos aos restaurantes por peso ainda são escassos, não permitindo uma avaliação sistematizada acerca do tema.

\section{O mercado de alimentação fora de casa}

A tendência de aumento da alimentação fora de casa em regiões urbanas é observada mundialmente. No Brasil, essa relação aumentou de $24 \%$ para $31 \%$ em seis anos, entre $2002 / 03$ e 2008/0910

Diante do crescimento no consumo de refeições fora de casa, torna-se importante discutir não só a qualidade nutricional dos alimentos oferecidos, mas também modelos de serviços que possam estimular escolhas saudáveis nesse contexto.

O setor de alimentação fora de casa pode ser dividido em dois segmentos: a alimentação comercial e a alimentação coletiva ${ }^{11}$. São exemplos de Unidades Produtoras de Refeições (UPR) comerciais: os restaurantes self-service (auto-serviço), restaurantes fast-food (redes de comida rápida), pratos prontos, restaurantes à la carte, bares e lanchonetes. Dentre eles, segundo a Associação Brasileira de Indústrias e Alimentação $(A B \mid A)^{12}$, os restaurantes comerciais, nas suas di- 
versas modalidades, são os que compreendem o maior faturamento do setor. Da mesma forma, nos Estados Unidos, os restaurantes fast-food e full-service (serviço completo) são as modalidades que detêm o maior volume de vendas no mercado $^{13}$. De acordo com a classificação adotada por Stewart et al. ${ }^{13}$, restaurantes fast-food são aqueles que oferecem um serviço rápido, de conveniência, enquanto restaurantes full-service tendem a apresentar cardápios mais variados e serviços de mesa mais completos.

No Brasil, os "restaurantes self-service ou de comida a quilo", de acordo com a Classificação Nacional de Atividades Econômicas (CNAE/IBGE) ${ }^{14}$, estão enquadrados na categoria "restaurantes e estabelecimentos de bebidas, com serviço completo".

Estudos sugerem que as refeições servidas nos restaurantes full-service são mais saudáveis do que as servidas nos restaurantes fast-food. Mehta \& Chand ${ }^{15}$ observaram que indivíduos que residiam em áreas norte-americanas com maior densidade de restaurantes fast-food do que full-service (medida pelo número de restaurantes por dez mil indivíduos) apresentaram IMC mais elevado. Corroborando, o estudo de Mellor et al. ${ }^{16}$ concluiu que estudantes que residem próximo a um restaurante fast-food apresentaram valores significativamente maiores do IMC, enquanto a proximidade de restaurantes full-service às residências não teve associação positiva significativa com IMC ou sobrepeso.

Estudo conduzido pelo United States Department of Agriculture (USDA) apresenta dados que revelam a dinâmica da distribuição do mercado norte-americano de alimentação fora de casa. Nesse país, as vendas dos restaurantes fast-food, que até a metade da década de 1990 superavam as dos restaurantes full-service, começaram a apresentar-se relativamente estagnadas e, a partir de 2002, os restaurantes full-service retomaram maior parte das vendas do mercado. Para os autores, esse quadro parece refletir a nova demanda dos consumidores, cada vez mais preocupados com a qualidade de vida e com a alimentação saudável. Destacam ainda que, embora diversos fatores possam contribuir para mudanças na composição do mercado de alimentação fora de casa, as demandas dos consumidores parecem ser as principais forças a motivar alterações nessa dinâmica ${ }^{13}$.

Sendo assim, conforme previsão da USDA, as despesas do consumidor com alimentação fora de casa continuarão a crescer, tanto nos restaurantes full-service, quanto nas redes de fast-food. Contudo, o aumento maior ocorrerá provavelmente nos restaurantes full-service. Isso indica, segundo os autores, que, conquanto os consumidores valorizem a conveniência, eles também exigirão refeições mais variadas, comodidade e entretenimento ${ }^{13}$.

\section{Fatores determinantes da escolha alimentar}

Embora as pessoas pareçam estar mais preocupadas com a qualidade de vida, no momento das escolhas alimentares outros fatores podem competir com a vontade de comer de forma saudável. É reconhecido que os comportamentos e as escolhas alimentares dos indivíduos são complexos e estão condicionados a diversos fatores.

Jomori et al. ${ }^{17}$, em artigo de revisão sobre determinantes da escolha alimentar, citam o preço, o sabor, a variedade, o valor nutricional, a aparência e a higiene como variáveis relacionadas ao alimento. Destacam, ainda, os fatores relacionados ao indivíduo, que incluem variáveis biológicas, socioculturais, antropológicas e econômicas.

Para Story et al. ${ }^{18}$, os fatores individuais relacionados à escolha alimentar incluem aspectos cognitivos, comportamentais, biológicos e demográficos. Os contextos ambientais abrangem ambientes sociais, ambientes físicos e macroambientes. O ambiente social compreende as interações com família, amigos, colegas e outros membros da comunidade. O ambiente físico diz respeito aos diversos locais onde as pessoas podem se 
alimentar ou comprar alimentos, como a própria casa, locais de trabalho, escolas, restaurantes e supermercados. E o macroambiente corresponde à comercialização de alimentos, normas sociais, sistemas de produção e distribuição de alimentos, bem como a políticas agrícolas e a estruturas econômicas.

Estudos têm demonstrado que o sabor, o valor monetário e o valor nutricional são as três dimensões principais relacionadas à escolha dos alimentos ${ }^{19,20}$. Apesar disso, segundo French ${ }^{21}$, mesmo que, em geral, as pessoas possam ter conhecimentos sobre escolhas alimentares saudáveis quando levam em consideração outras variáveis, é provável que optem pelo mais barato e mais saboroso, porém menos nutritivo. Assim, como o valor nutricional dos alimentos parece apresentar menor relevância do que o preço, estratégias de redução de preço podem ser uma boa forma para estimular a promoção de hábitos alimentares saudáveis ${ }^{21,22}$.

Essas variáveis relacionadas à escolha alimentar também se destacaram em pesquisa realizada com trabalhadores norte-americanos. No entanto, para a maioria dos indivíduos da referida pesquisa (34\%), o fator mais importante para a escolha dos alimentos para o almoço foi a conveniência, seguida pelo sabor (28\%), custo (21\%) e saúde (17\%). O estudo revelou ainda que a importância dada a cada uma dessas variáveis é diferente para indivíduos pertencentes a distintos grupos sociodemográficos. Para os trabalhadores mais velhos, conveniência e saúde foram citadas como os fatores que mais influenciavam a escolha alimentar, enquanto para os indivíduos mais jovens, o preço dos alimentos é aspecto primordial no momento da escolha, seguido de conveniência e sabor. Indivíduos com maior escolaridade declararam que o preço é importante na escolha dos alimentos com menos frequência do que os com menor escolaridade 23 .

Outros estudos mostram a influência das características sociodemográficas na escolha de alimentos saudáveis ${ }^{24-26}$. Há indícios de que o consumo de frutas e de vegetais seja maior entre as mulheres do que entre os homens ${ }^{24-26}$, entre pessoas com maior escolaridade ${ }^{26}$ e indivíduos pertencentes a faixas etárias mais elevadas ${ }^{24}$.

Embora as pesquisas sobre os determinantes de comportamentos alimentares em adultos, adolescentes e crianças tenham sido predominantemente centradas na influência dos fatores individuais ${ }^{27}$, parece estar havendo uma mudança, com atenção voltada para os determinantes ambientais da escolha alimentar. No entanto, pesquisas com esse enfoque ou sobre como fatores ambientais podem interagir com fatores individuais e sobre intervenções viáveis e eficazes para meIhorar os ambientes alimentares ainda são escassas $^{28,29}$.

Na Dinamarca, Lassen et al. ${ }^{30}$ avaliaram o impacto de duas modalidades de distribuição de refeições na escolha alimentar de 180 comensais que almoçavam em restaurantes localizados no ambiente de trabalho. Observaram maior consumo de frutas e vegetais e menor consumo de alimentos de alta densidade energética por aqueles que frequentavam restaurantes com sistema de bufê em comparação com os que almoçavam em restaurantes à la carte. Nos restaurantes com serviço de bufê, o padrão de consumo de frutas e vegetais foi semelhante para ambos os sexos, apesar de relatos científicos de que os homens consomem menos esses alimentos do que as muIheres $^{24}$. Os dados do estudo dinamarquês sugerem que diferentes modalidades de distribuição de refeições podem ocasionar diferenças no consumo de alimentos mais saudáveis.

Outro estudo ${ }^{31}$ envolveu cinco unidades produtoras de refeições situadas no ambiente de trabalho. Um estabelecimento oferecia serviço de bufê, com uma variedade de alimentos por um preço fixo, e os demais apresentavam um sistema denominado "cash cafeteria", em que o comensal escolhia e pagava por cada item selecionado. Objetivou-se investigar se mudanças significativas no consumo de Frutas e Vegetais (F\&V) eram possíveis de serem alcançadas após intervenção com ênfase em tornar tais alimentos mais atrativos, por meio de cortes e formas de preparo. A oferta 
de F\&V era feita na forma de "pratos quentes", "pratos frios", "saladas" e "frutas frescas + snacks à base de vegetais". Os dados iniciais do estudo mostraram que o estabelecimento com seviço de bufê já apresentava os maiores valores de consumo de F\&V por pessoa, quando comparado aos demais. Após a intervenção, houve aumento significativo no consumo de F\&V em todos os locais estudados. Destaca-se que, no decorrer do estudo, dois estabelecimentos mudaram a forma de distribuição para o sistema bufê. Em um deles, o consumo de F\&V continuou aumentando, especialmente na forma de saladas, enquanto no outro houve uma redução do consumo de F\&V, embora não significativa. Essa redução se deu principalmente pelo menor consumo de "pratos frios" e por não serem mais disponibilizadas "frutas frescas + snacks à base de vegetais". No entanto, o consumo de saladas se manteve. Para os autores, parece ser mais fácil que os comensais escolham saladas quando elas compõem um bufê do que quando é preciso pagar por elas separadamente.

No Brasil, o já citado estudo de Bezerra \& Sichierí investigou a relação entre consumo de refeições fora de casa e sobrepeso e obesidade entre adultos, e observou que o consumo de refeições tradicionais ou "sit-down meals" é mais frequente do que fast-food. As refeições tradicionais estiveram positivamente associadas com sobrepeso e obesidade nos homens; porém, entre as mulheres, revelou-se um fator de proteção para o excesso de peso e obesidade, sugerindo, de acordo com as autoras, que as mulheres brasileiras fazem escolhas mais saudáveis quando realizam as refeições fora de casa.

Os restaurantes estão entre os parceiros preferenciais das iniciativas para implementação da Estratégia Global da Organização Mundial de Saúde para Alimentação Saudável, Atividade Física e Saúde ${ }^{32}$. Corroborando, para Glanz \& Hoelscher ${ }^{33}$, os restaurantes estão entre os locais mais importantes e promissores para o desenvolvimento de estratégias políticas, ambientais e de redução de preços para estimular o consumo de alimentos saudáveis, como frutas e vegetais. Todavia, o Departamento de Saúde e Serviços Humanos dos Estados Unidos da América ${ }^{34}$ destaca que escolhas alimentares saudáveis somente podem ocorrer se houver ambientes favoráveis, com opções de alimentos saudáveis e com custo acessível.

\section{Restaurantes self-service por peso: este modelo brasileiro pode estimular escolhas saudáveis?}

Nos sistemas de distribuição em self-service ou autosserviço, os pratos quentes e frios são apresentados em balcões onde o cliente se serve com o seu prato, escolhendo entre as diversas opções oferecidas. Nessa modalidade de serviço, diferentemente do contexto familiar, no qual as decisões alimentares são, em grande parte, delegadas à dona de casa, o comensal decide, de maneira individual, o que ele irá consumir, realizando suas escolhas tendo por base uma diversidade de produtos, cuja estrutura e importância são definidas pelo responsável do restaurante ${ }^{35}$.

Os restaurantes de autosserviço parecem ter surgido para atender à necessidade de uma alimentação mais variada e rápida, na qual o consumidor assume uma parte do serviço, mas continua contando com as comodidades de serviço de mesa.

Em estudo realizado em restaurante self-service vinculado ao Programa de Alimentação do Trabalhador (PAT), observou-se que, quanto à adequação nutricional do cardápio oferecido pelo programa, tomando como base o consumo médio diário per capita, o almoço consumido foi hipoglicídico, hiperproteico e hiperlipídico para homens e mulheres e normoenergético para os homens, mas hiperenergético para as mulheres ${ }^{36}$. Do mesmo modo, Savio et al. ${ }^{37}$, em pesquisa envolvendo cinquenta restaurantes self-service filiados ao PAT, observaram que $43 \%$ da população estudada apresentaram excesso de peso. Ressalta-se que restaurantes coletivos ligados ao PAT normalmente oferecem menor 
variedade de escolhas do que os restaurantes comerciais por peso. Stolte et al. ${ }^{38}$ e Vanin et al. ${ }^{39}$ encontraram, em restaurantes de bufê ligados ao PAT, respectivamente, cardápios compostos por (a) arroz, feijão, um tipo de carne porcionada, um tipo de guarnição e uma salada e (b) arroz, feijão, um prato principal, uma opção de ovos, duas guarnições, três tipos de saladas, uma sobremesa ou uma opção de fruta. Entretanto, estudo realizado por Santos ${ }^{40}$ constatou que um restaurante self-service por peso comercial ofertava 55 variedades de preparações, sendo 25 compostas prioritariamente por frutas ou vegetais.

Na realidade brasileira, a modalidade self-service inclui cada vez mais o sistema por peso, que se constitui um modelo no qual o comensal escolhe o que deseja consumir, pagando o equivalente ao peso das preparações culinárias colocadas em seu prato. Embora haja, ainda, poucas pesquisas científicas sobre os restaurantes por peso, a modalidade difundiu-se por todo o país, independentemente de região ou de tipo de restaurante.

A denominação mais comum para tais locais é restaurante "por quilo". Entretanto, Magnée ${ }^{41}$ enfatiza a importância de denominá-los por peso, pois o quilo é uma unidade de medida que, dificilmente, o comensal alcançará individualmente em comida.

Inicialmente, esses restaurantes atraíam muitos comensais pelo fato de a comida oferecida possuir um caráter mais caseiro quando comparada a outros tipos de restaurantes, como os fast-food $^{42}$. Entretanto, pode-se verificar atualmente alimentos e preparações nesse tipo de estabelecimento que não são, necessariamente, parecidos com a alimentação disponível em casa.

Em virtude de suas características, os restaurantes por peso oferecem, além de rapidez e praticidade, a possibilidade de uma refeição completa, variada e com um custo acessível, quando comparados com algumas outras opções de serviços em alimentação fora de casa ${ }^{43}$. Acredita-se, ainda, que a possibilidade de o comensal compor seu prato de acordo com suas preferências e a variedade de oferta permitam escolhas saudáveis.

Ainda que não tenham sido encontrados estudos que confirmem que os sistemas de bufê self-service levem as pessoas a cometerem excessos, resultando no consumo de maiores porções, esse pensamento empírico pode ser contrastado pelos dados do já citado estudo de Lassen ${ }^{31}$, no qual foram encontrados indícios de que esse tipo de distribuição de refeições parece estimular a escolha de frutas e vegetais, especialmente na forma de saladas. Assim, mesmo que a hipótese de maior consumo fosse comprovada, parece que escolhas mais saudáveis estariam compondo o prato do comensal. A possibilidade de excessos em restaurantes self-service possivelmente seria reduzida em restaurantes por peso, visto que o preço a ser pago pela refeição é determinado pelo peso do prato. Dessa forma, somando essa questão com as outras características dessa modalidade de serviço anteriormente discutidas, os restaurantes self-service por peso podem ser uma importante alternativa para alimentação saudável fora de casa. Contudo, outros estudos precisam ser realizados para confirmar tal hipótese.

No estudo de Jomori26, que investigou a escolha alimentar de comensais em um restaurante por peso coletivo, o maior número de comensais escolheu no restaurante aquelas preparações que lhes remetessem ao ambiente doméstico e à estrutura tradicional de refeição. Outro estudo, que avaliou a composição dos pratos de 560 indivíduos com idade entre 16 e 59 anos que almoçavam em restaurante por peso comercial, concluiu que $82 \%$ dos comensais escolheram pelo menos um tipo de fruta ou vegetal para compor a refeição do almoço, sem diferença significativa quanto ao sexo, faixa etária, estado civil e escolaridade ${ }^{40}$. Esse dado é semeIhante ao encontrado no já mencionado estudo de Lassen et al. ${ }^{30}$, no qual homens e mulheres que almoçavam em restaurantes com serviço de bufê dinamarqueses apresentaram elevado consumo desses alimentos, sem diferença significativa entre os referidos grupos. Desse modo, reforça- 
-se a hipótese de que sistemas de bufê que oferecem variedade de alimentos com opções saudáveis de escolha parecem ser uma estratégia promissora para estimular escolhas alimentares saudáveis nas refeições realizadas fora de casa.

Vale ressaltar que, para que sejam fornecidas refeições nutricionalmente adequadas nesse tipo de restaurante, o nutricionista apresenta papel de destaque. Segundo o Conselho Federal de Nutricionistas, são atribuiç̧ões desses profissionais o planejamento e a elaboração dos cardápios e a coordenação do desenvolvimento de receituários e fichas técnicas ${ }^{44}$. Em restaurantes self-service, a promoção de saúde por meio de tais atribuições é um desafio ainda maior, visto que o comensal é quem realiza as escolhas, podendo fazê-las de maneira inadequada. Assim, destaca-se essa atuação tanto na disponibilização de preparações saudáveis quanto na promoção de programas de educação alimentar e nutricional, outra atribuição prevista pelo CFN.

\section{CONSIDERAÇÕES FINAIS}

Levando-se em conta o aumento do consumo de refeições fora de casa e a complexidade dos fatores que envolvem os comportamentos alimentares dos indivíduos, julga-se necessária a realização de novas pesquisas acerca da influência dos fatores ambientais nas escolhas dos alimentos. Além disso, considera-se de extrema necessidade a criação de ambientes favoráveis à saúde, que possibilitem o acesso a alimentos saudáveis, com destaque para atuação do nutricionista para a efetiva disponibilidade de opções de alimentos saudáveis.

Acredita-se que os restaurantes por peso, por reunirem características como a variedade de escolhas, a possibilidade da prática de preços acessíveis, a rapidez e a conveniência, possam representar uma alternativa de alimentação saudável fora de casa. Porém, são necessárias intervenções na forma de campanhas de saúde pública para educação sobre alimentação saudável, criação de medidas de incentivo aos estabelecimentos para que ofereçam opções saudáveis e para a disponibilização de informações alimentares e nutricionais das preparações oferecidas.

\section{COLABORADORES}

Todos os autores participaram da concepção e da redação do artigo.

\section{REFERÊ NCIAS}

1. Diez Garcia RW. Reflexos da globalização na cultura alimentar: considerações sobre as mudanças na alimentação urbana. Rev Nutr. 2003; 16(4):483-92. doi: 10.1590/S1415-52732003000400011.

2. Duffey KJ, Gordon-Larsen P, Jacobs DR Jr, Williams OD, Popkin BM. Differential associations of fast food and restaurant food consumption with 3-y change in body mass index: the Coronary Artery Risk Development in Young Adults Study. Am J Clin Nutr. 2007; 85(1):201-8.

3. Jeffery RW, Baxter J, McGuire M, Linde J. Are fast food restaurants an environmental risk factor for obesity? Int J Behav Nutr Phys Act. 2006; 3(1):2.

4. Paeratakul $S$, Ferdinand DP, Champagne $C M$, Ryan DH, Bray GA. Fast-food consumption among US adults and children: dietary and nutrient intake profile. J Am Diet Assoc. 2003; 103(10):1332-8.

5. Guthrie JF, Lin BH, Frazao E. Role of food prepared away from home in the American diet, 1977-78 versus 1994-96: changes and consequences. J Nutr Educ Behav. 2002; 34(3):140-50.

6. Bezerra IN, Sichieri R. Eating out of home and obesity: a Brazilian nationwide survey. Public Health Nutr. 2009; 12(11):2037-43.

7. Instituto Brasileiro de Geografia e Estatística. Pesquisa de Orçamentos Familiares - 2002-2003: aquisição alimentar domiciliar per capita, Brasil e grandes regiões. Rio de Janeiro: IBGE; 2004.

8. Kant AK, Graubard BI. Eating out in America, 1987-2000: trends and nutritional correlates. Prev Med. 2004; 38(2):243-9.

9. Ma Y, Bertone ER, Stanek EJ, Reed GW, Herbert $J R$, Cohen NL, et al. Association between eating patterns and obesity in a free-living adult population. Am J Epidemiol. 2003; 158(1):85-92.

10. Instituto Brasileiro de Geografia e Estatística. Pesquisa de Orçamentos Familiares 2008-2009: despesas, rendimentos e condições de vida. Rio de Janeiro: IBGE; 2010. 
11. Proença RPC. Inovação tecnológica na produção de alimentação coletiva. $2^{a}$ ed. Florianópolis: Editora Insular; 2000.

12. Associação Brasileira de Indústrias e Alimentação. Balanço anual 2005 e perspectivas para 2006. [acesso 2007 out 8]. Disponível em: <http://www. abia.org.br/anexos/BalancoAnual2005.pdf>.

13. Stewart H, Blisard N, Bhuyan S, Nayga RMJr. United States Department of Agriculture. USDA. The demand for food away from home: full-service or fast food? 2004. [cited 2007 Oct. 8]. Available from: <http://www.ers.usda.gov>.

14. Instituto Brasileiro de Geografia e Estatística. Classificação nacional de atividades econômicas: Versão 1.0. $2^{\text {a }}$ ed. Rio de Janeiro: IBGE; 2004 [acesso 2011 fev. 10]. Disponível em: <http://www.ibge.gov.br>.

15. Mehta NK, Chang VW. Weight status and restaurant availability a multilevel analysis. Am J Prev Med. 2008; 34(2):127-33.

16. Mellor JM, Dolan CB, Rapoport RB. Child body mass index, obesity, and proximity to fast food restaurants. Int J Pediatr Obes. 2010; 6(1):60-8.

17. Jomori MM, Proenca RPC, Calvo MCM. Food choice factors. Rev Nutr. 2008; 21(1):63-73. doi: 10.1590/S1 415-52732008000100007.

18. Story M, Kaphingst KM, Robinson-O'Brien R, Glanz $K$. Creating healthy food and eating environments: policy and environmental approaches. Ann Rev Public Health. 2008; 29(1):253-72.

19. Cassady D, Jetter KM, Culp J, Is price a barrier to eating more fruit and vegetables for low-income families? J Am Diet Assoc. 2007; 107(11):1909-15.

20. Glanz K, Basil M, Maibach E, Goldberg J, Snyder $D$. Why Americans eat what they do: taste, nutrition, cost, convenience, and weight control concerns as influences on food consumption. J Am Diet Assoc. 1998; 98(10):1118-26.

21. French SA. Pricing effects on food choices. J Nutr. 2003; 133(3):841S-43S.

22. Han S, Gupta S, Lechmann DR. Consumer price sensitivity and price thresholds. J Retail. 2001; 77(4):435-56.

23. Blanck HM, Yaroch AL, Atienza AA, Yi SL, Zhang J, Mâsse LC. Factors influencing lunchtime food choices among working Americans. Health Educ Behav. 2009; 36(2):289-301.

24. Blanck HM, Gillespie C, Kimmons JE, Seymour JD, Serdula, MK. Trends in fruit and vegetable consumption among U.S. men and women, 1994-2005. Prev Chronic Dis. 2008; 5(2):1-10.

25. Australian Bureau of Statistics National Health Survey: summary of results, Australia 2004-05. Canberra; 2006 [cited 2007 Oct. 18]. Available from: <http://www.ausstats.abs.gov.au>.
26. Jomori MM. Escolha alimentar do comensal de um restaurante por peso [mestrado]. Florianópolis: Universidade Federal de Santa Catarina; 2006.

27. Brug J, van Lenthe F, editors. Environmental determinants and interventions for physical activity, nutrition and smoking: a review. Zoetermeer: Speed-Print; 2005. p.378-89.

28. Kremers SP, Bruijn GJ, Visscher TL, van Mechelen W, Vries NK, Brug J. Environmental influences on energy balance-related behaviors: a dual-process view. Int J Behav Nutr Phys Act. 2006; 3(1):9.

29. Ball K, Timperio A, Crawford D. Understanding environmental influences on nutrition and physical activity behaviors: where should we look and what should we count? Int J Behav Nutr Phys Act. 2006; 3(1):33.

30. Lassen A, Hansen K, Trolle E. Comparison of buffet and à la carte serving at worksite canteens on nutrient intake and fruit and vegetable consumption. Public Health Nutrition. 2007; 10(3):292-7.

31. Lassen A, Thorsen AV, Trolle E, Elsig M, Ovesen L. Successful strategies to increase the consumption of fruits and vegetables: results from the Danish '6 a day' Work-site Canteen Model Study. Public Health Nutr. 2004; 7(2):263-70.

32. World Health Organization. Global strategy on diet, physical activity and health: list of all documents and publications. Geneva: WHO; 2004 [cited 2009 Mar 15]. Available from: <http://www.who.int/ dietphysicalactivity/strategy/eb11344/strategy_ english_web.pdf>.

33. Glanz K, Hoelscher D. Increasing fruit and vegetable intake by changing environments, policy and pricing: restaurant-based research, strategies, and recommendations. Prev Med. 2004; 39 (Suppl 2): S88-93.

34. US Department of Health and Human Services. The Surgeon General's call to action to prevent and decrease overweight and obesity. Rockville (MD): US Department of Health and Human Services; 2001.

35. Poulain J-P. Sociologias da alimentação. Florianópolis: UFSC; 2004. Série Nutrição.

36. Amorim MMA, Junqueira RG, Jokl L. Adequação nutricional do almoço self-service de uma empresa de Santa Luzia, MG. Rev Nutr. 2005; 18(1):145-56. doi: 10.1590/S1415-52732005000100013.

37. Savio KEO, Costa THM, Miazaki E, Schmitz BAS. Avaliação do almoço servido a participantes do Programa de Alimentação do Trabalhador. Rev Saúde Pública. 2005; 39(2):148-55. doi: 10.1590/\$0034-8 9102005000200002. 
38. Stolte D, Hennington EA, Bernardes JS. Sentidos da alimentação e da saúde: contribuições para a análise do programa de alimentação do trabalhador. Cad Saúde Pública. 2006; 22(9):1915-24. doi: 10.1590/S0102-311X2006000900023.

39. Vanin M, Southier N, Novello D, Francischetti VA. Adequação nutricional do almoço de uma unidade de alimentação e nutrição de Guarapuava - PR. Rev Salus. 2007; 1(1):31-8.

40. Santos MV. Características sócio-demográficas e componentes alimentares dos pratos de comensais em restaurantes por peso [mestrado]. Florianópolis: Universidade Federal de Santa Catarina; 2009.

41. Magnée HM. Manual do self-service. São Paulo: Varela; 1996.
42. Abdala MC. Novos hábitos alimentares e formas de sociabilidade de famílias mineiras. Cad Espaço Fem. 2001; 8(9)89-106.

43. Abreu ES, Torres EAFS. Restaurante "por quilo": vale o quanto pesa? Uma avaliação do padrão alimentar em restaurantes em São Paulo, SP. Nutrire. 2003; 25(1):7-22.

44. Conselho Federal de Nutricionistas. Resolução CFN $N^{\circ}$ 380/2005. [acesso 2011 jan 20]. Disponível em: <http://www.cfn.org.br/novosite/pdf/res/2005/ res380.pdf>.

Recebido em: 11/1/2010

Versão final reapresentada em: 25/2/2011

Aprovado em: 13/5/2011 